\title{
Composite bound states of wide and narrow envelope solitons in the coupled Schrödinger equations through matched asymptotic expansions
}

\author{
John P Boyd $\dagger$ and Benkui Tan $\ddagger$ \\ $\dagger$ Department of Atmospheric, Oceanic and Space Science, University of Michigan, 2455 \\ Hayward Avenue, Ann Arbor, MI 48109, USA \\ \$ Department of Geophysics, Peking University, Beijing 100871, People’s Republic of China \\ E-mail: jpboyd@engin.umich.edu and dqgchw@ibmstone.pku.edu.cn
}

Received 9 September 1998, in final form 25 May 1999

Recommended by Y Kuramoto

\begin{abstract}
Coupled nonlinear Schrödinger equations, linked by cross modulation terms, arise in both nonlinear optics and in Rossby waves in the atmosphere and ocean. Numerically, Akhmediev and Ankiewicz and Haelterman and Sheppard discovered a class of soiltary waves which are composed of a tall, narrow sech-shaped soliton in one mode, bound to a pair of short, wide sechshaped peaks in the other mode or polarization. Through the method of matched asymptotic expansions, we derive analytical approximations to these solitary waves, and to their periodic generalization, which have been hitherto accessible only through numerical computation.
\end{abstract}

AMS classification scheme numbers: 76B25, 76C20, 35B25

\section{Introduction}

The nonlinear Schrödinger (NLS) equation has been derived as a model for weakly nonlinear wavepackets in a wide variety of physical systems by means of the perturbative method of multiple scales. When there are resonances between different wave modes or for birefringent fibres in nonlinear optics, the NLS equation must be replaced by a coupled pair of equations which have the form

$$
\begin{aligned}
& \mathrm{i} A_{1, t}+\alpha_{1} A_{1, x x}+\left(\sigma_{1}\left|A_{1}\right|^{2}+v_{12}\left|A_{2}\right|^{2}\right) A_{1}=0 \\
& \mathrm{i} A_{2, t}+\alpha_{2} A_{2, x x}+\left(\sigma_{2}\left|A_{2}\right|^{2}+v_{21}\left|A_{1}\right|^{2}\right) A_{2}=0
\end{aligned}
$$

where $A_{1}$ and $A_{2}$ are the amplitudes of the wave envelope in the different modes (or polarizations). We shall call this the CNLS (coupled-nonlinear Schrödinger) system. The coefficients $\alpha_{1}$ and $\alpha_{2}$ are the so-called 'dispersion coefficients', $\sigma_{1}$ and $\sigma_{2}$ are the 'Landau constants' describing the self-modulation of the wavepackets, and $\nu_{12}$ and $v_{21}$ are the 'coupling constants' of the cross-modulation between the two wavepackets.

Tan and Liu [19] and Tan [17] have derived this system for applications in geophysical fluid dynamics. The CNLS has also been extensively studied in nonlinear optics where only a partial list of references includes [1,10-13, 15, 20,21,23-28]. 
We shall leave further discussion of the physics and of general time-dependent solutions to the references. Our goal here is to understand solitary wave solutions of the form

$$
A_{1}=\exp \left(\mathrm{i} \mu^{2} \alpha_{1} t\right) u(x), \quad A_{2}=\exp \left(\mathrm{i} \beta^{2} \alpha_{2} t\right) v(x)
$$

where $\mu, \beta$ and $u, v$ are assumed to be real. The time-dependent CNLS equations simplify to a set of two coupled ordinary differential equations (ODEs) which we shall dub the steadilyoscillating coupled-nonlinear Schrödinger (SOCNLS) equations:

$$
\begin{aligned}
& u_{x x}-\mu^{2} u+\frac{\sigma_{1}}{\alpha_{1}} u^{3}+\frac{\nu_{12}}{\alpha_{1}} u v^{2}=0 \\
& v_{x x}-\beta^{2} v+\frac{\sigma_{2}}{\alpha_{2}} v^{3}+\frac{\nu_{21}}{\alpha_{2}} u^{2} v=0 .
\end{aligned}
$$

The SOCNLS system admits many branches of solutions. Our interest is a branch independently discovered by Akhmediev and Ankiewicz [2] and Haelterman and Sheppard [10]. The former called them 'B-type' solitons; the latter had no special name. We shall dub these solutions 'composite multiple-scale' solitons because when $\mu \gg \beta$, these consist of a tall, narrow sech-like peak in $u$ which binds together two short, broad peaks in $v$. Because, for this species of solitary wave, both ODEs cannot be approximated by linear equations even in the limit $\beta / \mu \rightarrow 0$, Akhmediev and Ankiewicz and Haelterman and Sheppard were unable to find analytical approximations and were pessimistic that this would ever be possible.

In this paper, we show that by using the method of matched asymptotic expansions, it is possible to obtain analytical approximations to such composite, multiple-scaled solitons in the limit that the parameter

$$
\epsilon \equiv \beta / \mu
$$

is small where $\epsilon$ is the ratio of the two frequencies at which the different components $A_{1}$ and $A_{2}$ oscillate in time. These solitons and the 'inner' and 'outer' regions of the matched asymptotic method are illustrated schematically in figure 1.

One important simplification is that when $\epsilon \ll 1, v \sim \mathrm{O}(\epsilon)$ and this component is only an $\mathrm{O}\left(\epsilon^{2}\right)$ perturbation of the first equation of the SOCNLS system. This implies that if we only wish to work to lowest nontrivial order, it is sufficient to write

$$
u \sim \sqrt{2 \frac{\alpha_{1}}{\sigma_{1}}} \mu \operatorname{sech}(\mu x)+\mathrm{O}\left(\epsilon^{2}\right)
$$

and solve a single, uncoupled $O D E$ for $v$ alone. By introducing the new coordinate

$$
y \equiv \mu x \leftrightarrow \frac{\mathrm{d}}{\mathrm{d} x}=\mu \frac{\mathrm{d}}{\mathrm{d} y}
$$

the new parameter

$$
\Xi \equiv 2 \frac{\nu_{21}}{\sigma_{1}} \frac{\alpha_{1}}{\alpha_{2}}
$$

and the new unknown

$$
w=\sqrt{\frac{\sigma_{2}}{2 \alpha_{2} \mu^{2}}} v
$$

the single ODE for $v$ becomes what we shall dub the 'reduced problem':

$$
w_{y y}-\epsilon^{2} w+2 w^{3}+\Xi \operatorname{sech}^{2}(y) w=0
$$

Since the numerical studies by Akhmediev and Ankiewicz and Haelterman and Sheppard have found only symmetric solutions, we shall restrict our attention to solutions which are symmetric 


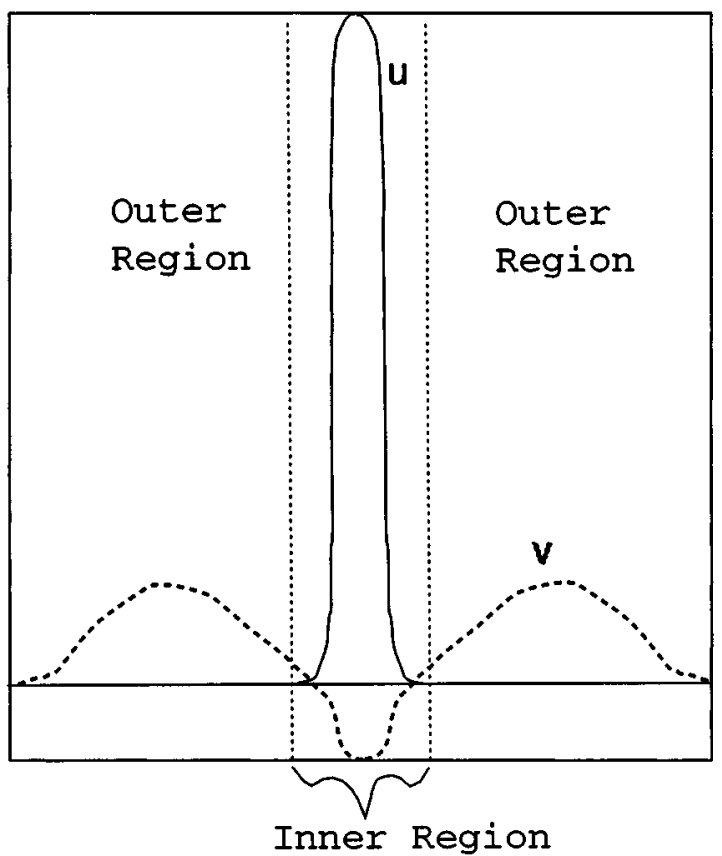

Figure 1. A schematic of the composite solitary waves which are studied in this paper. A tall, narrow $u$ component (solid) is bound to a pair of short, wide peaks in the $v$ component (thick dashed curve). The thin vertical dotted lines separate the inner region.

with respect to $y=0$, which implies that the first derivative of $w$ at the origin is zero. The reduced problem is then completely specified by adding the initial conditions:

$$
w(0)=w_{0}, \quad w_{y}(0)=0 .
$$

The primary goal of our paper is to solve this ODE through matched asympotics to lowest order. This will simultaneously generate approximations to the SOCNLS system to lowest and first order. (At $\mathrm{O}\left(\epsilon^{2}\right), v$ will modify $u$ from the sech function in equation (7) and we must explicitly attack the SOCNLS as a coupled system to approximate its solution, but such second- and higher-order approximations are beyond the scope of this paper.)

Our analysis is formal; we do not prove that $(u, v)$ can be asymptotically approximated by power series in $\epsilon$ to all orders. We also do not prove that a solution exists. One of us has written a monograph [7] on so-called 'weakly nonlocal' solitary waves, which flunk the classical definition of a solitary wave because of terms that are exponentially small in $1 / \epsilon$, and so lie beyond all orders in an $\epsilon$-power series. However, there is no evidence that the SOCNLS solitary waves are 'weakly nonlocal'. The highly accurate numerical solutions presented below strongly suggest that our lowest-order approximation, which certainly is self-consistent at lowest order, is telling a truthful story about the physics, whatever logarithms in $\epsilon$ or other adventures may arise at higher order [9].

The matched asymptotic analysis will come in two varieties. First, the general solution to the reduced problem is a three-parameter family where the parameters are $\left(\epsilon, \Xi, w_{0}\right)$. We shall show how this can be solved by using elliptic functions. Second, there is a two-dimensional family of solutions which are solitary waves in the sense that $|w(y)| \rightarrow 0$ as $|y| \rightarrow \infty$. The solitons require some procedural modifications from the general solution. 


\section{An overview of matched asymptotics}

For this problem, the heart of matched asymptotics is that in the inner region, the nonlinear term is dropped but the sech-squared term is kept whereas in the outer region the sech-squared term is omitted but the nonlinear term is retained.

Because $\operatorname{sech}(y)$ varies on an $\mathrm{O}(1)$ length scale, this will also be the length scale for $w(y)$ in the inner region. The $\epsilon^{2} w$ term can therefore be neglected in the inner region. The lowest-order inner approximation solves

$$
w_{y y}^{\text {inner,lowest }}+\Xi \operatorname{sech}^{2}(y) w^{\text {inner,lowest }} \sim 0, \quad y \ll 1 / \epsilon .
$$

To match to the outer approximation, which applies when $|y| \sim \mathrm{O}(1 / \epsilon)$, we need the 'outer limit of the inner approximation', which is simply the limit of the inner solution as $y \rightarrow \infty$. (This outer limit is a valid approximation to the reduced problem (11) in the intermediate range $1 \ll y \ll 1 / \epsilon$.) For large $y$, the lowest-order inner equation asymptotes to the differential equation $w_{y y}^{\text {inner,lowest }} \sim 0$, whose solution is a linear function of $y$. It follows that the outer limit at lowest order is

$$
w^{\text {inner, lowest }} \sim \epsilon^{r}\left\{p_{r 0}+p_{r 1} y\right\} \quad y \gg 1 /|\log (\epsilon)|
$$

where $r$ is the order of the lowest order; both $r=1$ and $r=2$ will arise in the analysis below.

If $w$ in the inner region is no larger than its maximum in the outer region, which will be shown later to be $\mathrm{O}(\epsilon)$, then the first-order inner equation is

$$
w_{y y}^{\text {inner, first }}+\Xi \operatorname{sech}^{2}(y) w^{\text {inner, first }} \sim \epsilon^{2} w^{\text {inner,lowest }}-2\left(w^{\text {inner,lowest }}\right)^{3} .
$$

For large $y$, the right-hand side is a cubic polynomial in $y$, and one can show by substituting a polynomial with undetermined coefficients into the equation (where the $\operatorname{sech}^{2}(y)$ term is negligible) that $w^{\text {inner, first }}$ is a quintic polynomial in the outer limit. And so it goes: the outer limit at arbitrary order is of the form

$$
w^{\text {inner }} \sim \sum_{j=1}^{\infty} \sum_{k=0}^{k_{\max }(j)} p_{j k} \epsilon^{j} y^{k}, \quad y \gg 1 .
$$

This outer limit must match, order-by-order, to the inner limit of the outer approximation $\dagger$.

In the outer region where $y \gg 1 /|\log (\epsilon)|$, the $\operatorname{sech}^{2}(y)$ term is negligible. The outer equation is

$$
w_{y y}^{(\text {outer })}-\epsilon^{2} w^{(\text {outer })}+2\left(w^{(\text {outer })}\right)^{3}=0, \quad|y| \gg 1 .
$$

This can be simplified by introducing the new variables

$$
\begin{aligned}
& \xi \equiv \epsilon y \quad \text { ['outer coordinate'] } \\
& W \equiv w^{\text {outer }} / \epsilon
\end{aligned}
$$

which eliminate all explicit dependence on $\epsilon$ to yield

$$
W_{\xi \xi}-W+2 W^{3}=0 .
$$

We have omitted the label 'lowest' because the neglected term decays exponentially fast with $|y|$. Without approximation, but rewritten in terms of the outer coordinate and unknown, the reduced problem is

$$
\begin{aligned}
W_{\xi \xi}-W+2 W^{3} & =\frac{\Xi}{\epsilon^{2}} \operatorname{sech}^{2}\left(\frac{\xi}{\epsilon}\right) W \\
& \sim \frac{4 \Xi}{\epsilon^{2}} \exp \left(-2 \frac{\xi}{\epsilon}\right) W, \quad \epsilon \rightarrow 0, \quad \text { fixed } \xi .
\end{aligned}
$$

$\dagger$ For expository simplicity, we consider an arbitrary-order solution of the reduced problem only to explain the mechanics of matched asymptotics; to solve the coupled SOCNLS system to arbitary order, we would need a similar expansion for the $u$ component, too. 
It follows that the term which is neglected in the outer approximation is exponentially small in $1 / \epsilon$ for any fixed value of the outer coordinate $\xi$. This implies that this term is 'beyond all orders', that is, as $\epsilon \rightarrow 0$, $\exp (-$ constant $/ \epsilon)$ goes to zero faster than any finite power of $\epsilon$. This in turn implies that this term cannot be consistently included in the order-by-order perturbation analysis at any finite order $[3,7,14,16]$. Thus, the outer equation is $W_{\xi \xi}-W+2 W^{3}=0$ not merely to lowest order but to all orders.

In the next section, we show that the exact outer solution is an elliptic function. In so far as the matching is concerned, however, the exact outer solution is irrelevant. Only the inner limit of the outer solution is needed for matching. This is obtained by expanding the outer solution as a power series in $\epsilon$ for fixed $\xi$ and then taking the limit as $\xi \rightarrow 0$, which allows a Taylor series in $\xi$ as well:

$$
w^{\text {outer }} \sim \sum_{J=1}^{\infty} \sum_{K=0}^{\infty} q_{J K} \epsilon^{J} \xi^{K}, \quad \xi \ll 1 .
$$

One might suppose that because the outer approximation is an elliptic function to all orders, one could set $q_{J K}=0$ for all $J \neq 1$. However, there is a subtlety. The matching conditions at various orders in $\epsilon$ will alter the amplitude or the phase of the outer approximation, even though it is always an elliptic function.

The matching principle, described particularly well in Van Dyke's monograph [22], is that the inner limit of the outer approximation must match the outer limit of the inner approximation. This requires

$$
p_{j k}=q_{j-k, k} .
$$

The inner and outer approximations can be blended into a composite approximation which is uniformly valid over all $y$ by writing

$$
w^{\text {composite }} \sim w^{\text {inner }}+w^{\text {outer }}-\operatorname{outer} \operatorname{limit}\left(w^{\text {inner }}\right)
$$

where the inner and outer approximations are taken to comparable order so that the outer limit of the inner approximation, which is the third term, may be equally well computed as the inner limit of the outer approximation. Because the third term is the inner limit of the outer approximation, the second and third terms cancel in the inner region where $w^{\text {composite }} \sim w^{\text {inner }}$. Similarly, in the outer region, the first and third terms cancel so that the composite solution equals the outer approximation where $\xi \sim \mathrm{O}(1)$ or larger.

Although our study will be henceforth restricted to lowest order, there are still two cases that must be discussed separately.

First case: $q_{10}$ different from zero while lower-order terms in $\epsilon$ are zero.

In other words, the constant in the $\xi$ power series at $\mathrm{O}(\epsilon)$ is nonzero. The matching conditions are

$$
p_{10}=q_{10} \quad \text { and } \quad p_{11}=q_{01} .
$$

Since $q_{01}=0$ if the outer solution is $\mathrm{O}(\epsilon)$, we have a contradiction unless $p_{11}=0$. This implies

$$
w^{\text {inner }} \sim \epsilon\left\{p_{10}+0 y\right\} .
$$

Put in words, the first-order inner approximation must asymptote to a constant, not to a linear function of $y$. In general, this is possible only when the parameter $\Xi$ is equal to $n(n+1)$ where $n$ is a non-negative integer, or when $\Xi$ is within $O(\epsilon)$ of such a value. This is precisely the case when the outer approximation is a solitary wave.

Second case: $q_{10}=0$ and $q_{11} \neq 0$ or in other words, the outer solution has a 'near-zero' at $\xi=0$. 
We describe this requirement as 'near-zero' because it is not necessary that the outer approximation vanish to all orders at $\xi=0$, but only that it have a root within $\mathrm{O}(\epsilon)$ of $\xi=0$. When these conditions are met, the asymptote of the lowest-order inner approximation is free to be a general linear function of $y$. In this case, the outer approximation is the elliptic cn for values of the elliptic modulus less than one, which implies that $W$ does not decay exponentially as $|y| \rightarrow 0$ so that the nonlinear wave is periodic rather than a solitary wave.

\section{Exact solution of the outer equation}

\subsection{Theorem}

The solution to the cnoidal subproblem can be summarized as the following theorem.

Theorem 1 (outer approximation (scaled)). Define $W(Y ; \chi)$ as the solution to the following problem:

$$
W_{Y Y}-W+2 W^{3}=0
$$

subject to the initial conditions

$$
W_{Y}(0)=\chi, \quad W(0)=0 .
$$

The solution is

$$
W=k\left(1+4 \chi^{2}\right)^{1 / 4} \operatorname{cn}\left(\left(1+4 \chi^{2}\right)^{1 / 4} \mathrm{Y}-K ; k\right)
$$

where $\mathrm{cn}(u ; k)$ is the usual elliptic $\mathrm{cn}$ function which is periodic in $u$ with period $4 K$ where $K(k)$ is the complete elliptic integral and $k$ is the elliptic modulus, which is given in terms of the initial condition $\chi$ by

$$
k=\sqrt{\frac{1}{2}+\frac{1}{2} \frac{1}{\sqrt{1+4 \chi^{2}}} .}
$$

This solution is periodic in $Y$ with period

$$
\mathcal{P}=4 K(k) \frac{1}{\left(1+4 \chi^{2}\right)^{1 / 4}} .
$$

Theorem 2 (outer approximation (unscaled)). The solution to

$$
w_{y y}-\epsilon^{2} w+2 w^{3}=0
$$

subject to the initial conditions

$$
w(0)=0, \quad w_{y}(0)=\epsilon^{2} \chi
$$

is

$$
w=\epsilon W(\epsilon y ; \chi)
$$

where $W(\xi ; \chi)$ is the elliptic function defined in the previous theorem.

The first theorem is proved by specializing section D.2.2 of [7] to a unit coefficient of the undifferentiated term. This gives the 'dispersion relation' for the cn function

$$
\mathcal{P}^{2}=16 K^{2}(k)\left\{2 k^{2}-1\right\}
$$

where $\mathcal{P}$ is the spatial period of the cn function, $K$ is the elliptic integral, and $k$ is the elliptic modulus. The elliptic function itself is

$$
W=4 k \frac{K}{\mathcal{P}} \operatorname{cn}\left(4 \frac{K}{\mathcal{P}} \xi-K ; k\right) .
$$


The derivative identity [7]

$$
\frac{\mathrm{d} \mathrm{cn}}{\mathrm{d} U}=-\operatorname{sn} \mathrm{dn}
$$

is easily converted to differentiation with respect to $\xi$ and simplified using the known special values $\operatorname{sn}(-K ; k)=-1$ and $\operatorname{dn}(-K ; k)=\sqrt{1-k^{2}}$. Matching the derivative to its specified value $\chi$ gives a quadratic equation in $k^{2}$ which can be solved explicitly for the elliptic modulus $k$ in terms of $\chi$.

The second theorem follows through the trivial rescaling of unknowns and the coordinate already given in equations (18)-(20).

Note that the outer equation is given by equation (32) to all orders, so the elliptic function is the outer solution to within an error which is exponentially small in $1 / \epsilon$.

\section{Inner approximation: general theory}

It is convenient to introduce a normalized form for the inner equation via

$$
w^{\text {inner,lowest }} \equiv \epsilon^{r} \omega \Omega(y ; \Xi)
$$

where $\Omega$ solves

$$
\Omega_{y y}+\Xi \operatorname{sech}^{2}(y) \Omega=0
$$

plus the initial conditions

$$
\Omega(0)=1 \quad \text { and } \quad \Omega_{y}(0)=0 .
$$

The exponent $r$ and the constant $\omega$ will be determined later by matching. The advantage of introducing the new unknown $\Omega$ is that the $\Omega$-problem is completely specified and can therefore be analysed independent of the matching.

The change of coordinate

$$
z \equiv \tanh (y)
$$

together with the identities $\mathrm{d} / \mathrm{d} y=\left(1-z^{2}\right) \mathrm{d} / \mathrm{d} z$ and $\operatorname{sech}^{2}(y)=1-z^{2}$ convert equation (39) into the Legendre equation

$$
\left\{\left(1-z^{2}\right) \Omega_{z z}-2 z \Omega_{z}\right\}+v(v+1) \Omega=0
$$

where we have introduced a new parameter $v$ (not be confused with the cross-modulation coefficients $\nu_{21}, v_{12}$ in the CNLS system) by the equation

$$
\Xi=v(v+1) \text {. }
$$

The general solution is

$$
a P_{v}(z)+b Q_{v}(z)
$$

where $P_{v}$ and $Q_{v}$ are the usual associated Legendre functions of zero order (zero superscript in the usual notation) and where the constants $a$ and $b$ are determined by the initial conditions. The subscript $v$ need not be an integer, but when it is, the $P_{v}(z)$ are the ordinary Legendre polynomials.

For the special case of $v$ a non-negative integer, the Legendre equation can be solved explicitly:

$$
\begin{aligned}
& \Omega(y, \Xi=0)=1 \\
& \Omega(y, \Xi=2)=1-y \tanh (y) \\
& \Omega(y, \Xi=6)=-2+3 \operatorname{sech}^{2}(y) \\
& \Omega(y, \Xi=n(n+1))= \begin{cases}P_{n}(\tanh (y)) / P_{n}(0), & n=\text { even integer } \\
Q_{n}(\tanh (y)) / Q_{n}(0), & n=\text { odd integer. }\end{cases}
\end{aligned}
$$


For noninteger $v$, however, the conversion to the Legendre equation is useful mostly to show that a search for a simple, closed-form analytic solution is futile.

It follows for general $\Xi$, as needed when $w$ (or equivalently $v$ ) is periodic rather than exponentially decaying with increasing values of the spatial coordinate, the inner problem must be solved numerically as done in the next section. For solitary waves, however, we are in the happy situation that the inner problem can be solved analytically through expansions which are given in section 7 .

\section{Solving the inner problem for general $\Xi$ : numerical method}

As noted in the previous section, it is not possible to solve the inner problem for arbitrary $\Xi$ in simple closed form. In this section, we describe a very accurate numerical method that succeeds where analysis fails. The spectral algorithm computes $w^{\text {inner }}$ at all points $y$, which is essential if we wish to graph the composite matched asymptotic approximation for all values of the spatial coordinate as done below. However, only two pieces of information from this numerical solution enter into the matching conditions with the outer approximation: the coefficients of the constant and the term which is linear in $y$ in the large- $y$ asymptotic approximation of the inner solution $\Omega(y)$. The rational Chebyshev pseudospectral method with one or two special basis functions is very useful for our purposes because these two coefficients-needed-for-matching are computed directly as basis coefficients in the spectral representation.

The solution is expanded as

$$
\Omega(y)=\sum_{j=1}^{N-2} a_{j}\{\cos (2 j t(y))-1\}+a_{N-1}+a_{N} \log (2 \cosh (y))
$$

where

$$
t \equiv \operatorname{arccot}(y / L) .
$$

The map parameter $L$ is a user-choosable constant; a little experimentation showed that $L=2$ was a good choice for all $N$ and all $\Xi<25$. The change of coordinate is such that the Fourier cosine series in $t(y)$ becomes a series of rational functions in $y$. Because $\Omega$ is symmetric with respect to $y=0$, only cosine functions of even degree are needed. The $N$ th basis function asymptotes to a linear function of $y$ (plus terms exponentially small in $|y|$ ) as $y \rightarrow \infty$ so that $a_{N}$ is equal to the constant $p_{21}$ needed for matching. The rational basis functions are chosen to be the difference between the cosines and one so that each basis function, except the $(N-1)$ st, vanishes as $|y| \rightarrow \infty$. Therefore, $a_{N-1}$, the coefficient of the trivial basis function, the constant one, is equal to the constant $p_{10}$ in the outer limit of the inner approximation.

The rational Chebyshev pseudospectral method is described in $[5,8]$. The role of the cotangent change-of-coordinate in transforming logarithmic endpoint singularities in the coordinate $z$ into weakly singular or nonsingular behaviour in $y$ is discussed in [4]. Special basis functions are analysed in [6]. In our calculations, we employed only a single special basis function, $\log (2 \cosh (y))$. (The constant one is actually one of the rational Chebyshev functions.) As explained in [6], the Legendre equation, when written in $z$, has not only a leading singularity proportional to $\log \left(1-z^{2}\right)(=-2 \log (\cosh (y)))$ but also weaker singularities proportional to $\left(1-z^{2}\right)^{k} \log \left(1-z^{2}\right)$ for all positive integers $k$. The rate of convergence can be improved by adding additional basis functions proportional to these weaker singularities. However, we found that a modest number of Chebyshev functions $(N<40)$ and one special basis function was sufficient. 
In the pseudospectral algorithm, a residual function is defined by substituting the spectral series, with symbolic coefficients, into the differential equation. One then imposes the condition that $\Omega(0)=1$ and also that the residual should be zero at $N-1$ collocation points

$$
y_{j}=L \cot \left(\pi \frac{j}{2 N}\right), \quad j=1, \ldots,(N-1) .
$$

These $N$ conditions are equivalent to the matrix equation $M a=f$ where $\boldsymbol{a}$ is a column vector containing the unknown spectral coefficients, $f$ is a column vector of zeros except for the $N$ th (initial-condition-specifying) row where $f_{N}=1$ and the elements of $M$ are, denoting the basis functions by $\phi_{j}(y)$,

$$
M_{i j}= \begin{cases}\phi_{j, y y}\left(y_{i}\right)+\Xi \operatorname{sech}^{2}\left(y_{i}\right) \phi_{j}\left(y_{i}\right), & i=1,2, \ldots,(N-1) \\ \phi_{j}(0), & i=N .\end{cases}
$$

By using this pseudospectral scheme, it is easy to solve the Legendre equation and directly determine the constant and linear term in the large- $|y|$ asymptotics. Inverting a $40 \times 40$ matrix is not much of a task even for a personal computer. Figure 2 shows the results for the outer limit of the inner approximation:
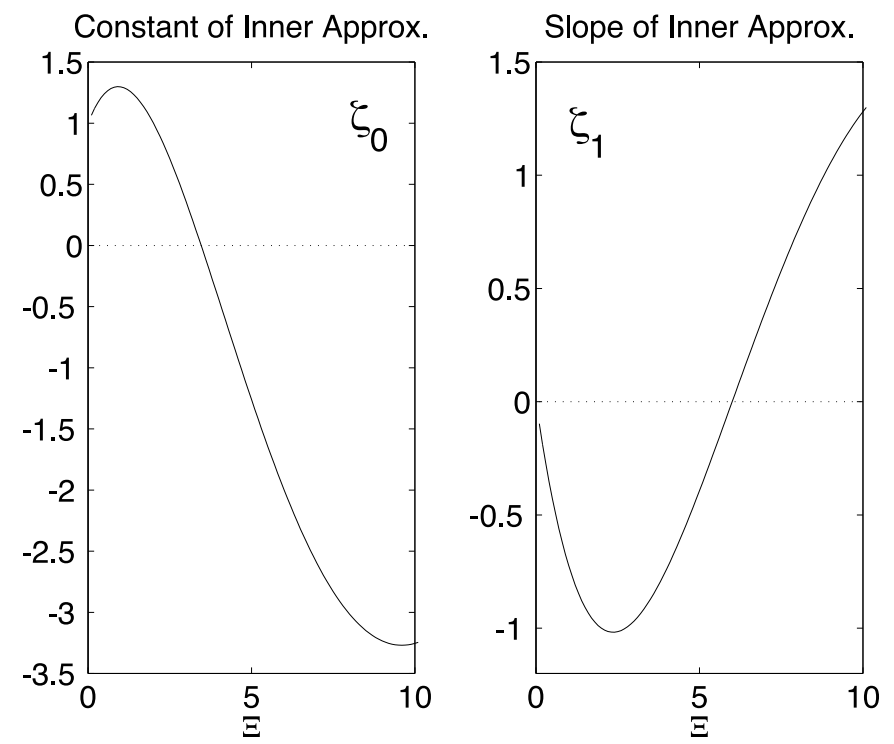

Figure 2. The outer limit of $\Omega(y ; \Xi)$ is a linear function of $y, \zeta_{0}+\zeta_{1} y$. This figure shows the constant $\zeta_{0}$ (left) and the coefficient of $y, \zeta_{1}$, (right). These were computed numerically using a rational Chebyshev basis supplemented with two special basis functions. Because the dependence of the inner approximation on the parameters $\epsilon$ and $w(0)$ is trivial (simple proportionalities), it is possible to express the inner approximation in terms of a rescaled function $\Omega$ whose outer limit is a function of $y$ and $\Xi$ only.

\section{Asymptotic matching, part I: general case (periodic waves)}

The general three-parameter family of solutions is specified by giving values for $(\Xi, \epsilon, w(0))$. However, to match to the outer solution, it turns out that $w(0)$ must be $\mathrm{O}\left(\epsilon^{2}\right)$ in general 
(although it is $\mathrm{O}(\epsilon)$ for the special case when the outer solution is a soliton). We therefore write

$$
w(0)=\epsilon^{2} \omega
$$

where $\omega \sim \mathrm{O}(1)$. Recalling that $\Omega(y)$ has been normalized to unit value at $y=0$, it follows that

$$
w^{\text {inner,lowest }}(y)=\omega \epsilon^{2} \Omega(y) .
$$

For arbitrary $\Xi$, the Legendre equation can be solved numerically to determine the outer limit of the inner approximation as

$$
w^{\text {inner, lowest }} \sim \epsilon^{2} \omega\left\{\zeta_{0}+\zeta_{1} y\right\}, \quad y \gg 1
$$

where the linear polynomial in braces is the asymptote of $\Omega(y ; \Xi)$. The coefficients $\zeta_{0}$ and $\zeta_{1}$, which are independent of $y$ but functions of the parameter $\Xi$, must be computed for a given $\Xi$ by using the rational Chebyshev pseudospectral method described in the previous section (see figure 2). Comparison with the symbolic power series, equation (16), shows that these coefficients can be written in terms of earlier symbols $p_{j k}$ as

$$
p_{20}=\omega \zeta_{0}(\Xi), \quad p_{21}=\omega \zeta_{1}(\Xi) \text {. }
$$

Matching to the terms of the double power series in $\xi$ and $\epsilon$ which is the inner approximation of the outer limit gives

$$
p_{10}=q_{10} ; \quad p_{20}=q_{20} ; \quad p_{21}=q_{11} .
$$

Since $p_{10}=0$, the first matching condition implies that $q_{10}=0$, too. However, we must have $q_{11} \neq 0$ so that it can match to $p_{21}$, which is nonzero in general. Put in words, this means that the $\mathrm{O}(\epsilon)$ terms in the inner limit of the outer approximation must be proportional to $\xi$, that is, to a linear polynomial with a zero constant. In other words, the outer solution must have a root to lowest order in the sense that the outer solution at $\xi=0$ must be $\mathrm{O}(\epsilon)$ smaller than its first derivative with respect to $\xi$ at the origin.

The outer equation has solutions that can be expressed in terms of either the dn function or the cn function, but the dn function has no real-valued zeros, so the outer solution must always be the cn function. It is possible that the origin does not exactly coincide with the root of the outer solution; in order that $q_{10}=0$ and $q_{11} \neq 0$, it is sufficient that the root be within $\mathrm{O}(\epsilon)$ of $\xi=0$. We can therefore write the outer solution, using the theorems of section 3 , as

$$
w^{\text {outer }}=\epsilon k\left(1+4 \chi^{2}\right)^{1 / 4} \operatorname{cn}\left(\left(1+4 \chi^{2}\right)^{1 / 4}(\xi-\epsilon \mathcal{S})-K ; k\right)
$$

where $\xi \equiv \epsilon y$ is the slow outer variable defined previously. The phase shift $\mathcal{S} \sim \mathrm{O}(1)$, which implies that $w(\xi=0) \sim \mathrm{O}\left(\epsilon^{2}\right)$, and not as large as $\mathrm{O}(\epsilon)$.

Strictly speaking, there are two outer approximations, one applicable left of the origin, and the other for positive $y$. Because $w(y)$ is symmetric, however, the left outer approximation is just the mirror image of the right outer approximation given analytically by equation (58):

$$
w^{\text {outer,left }}(y) \equiv w^{\text {outer, } \text {,ight }}(-y), \quad \forall y .
$$

Consequently, it is sufficient to discuss the outer approximation for $y>0$ and to match the inner and outer approximations for positive $y$ only. The symmetry ensures that the inner approximation will then also automatically match the outer approximation for negative $y$.

Equation (33) shows that

$$
w_{\xi}(0)=\epsilon \chi+\mathrm{O}\left(\epsilon^{2}\right)
$$

This implies that the linear coefficient of the inner limit of the outer solution is

$$
q_{11}=\chi
$$


The matching condition $p_{21}=q_{11}$ and equation (56) gives

$$
\chi=\omega \zeta_{1}(\Xi) \text {. }
$$

Put in words, the slope $\chi$ of the elliptic function at $\xi=0$, which completely determines the outer solution (except for the phase shift $\mathcal{S}$, which is higher order), is the product of $w(0) / \epsilon^{2}$ multiplied by the linear coefficient $\zeta_{1}(\Xi)$ of $\Omega(y ; \Xi)$.

A one-term Taylor approximation of the outer solution gives

$$
\begin{aligned}
w^{\text {outer }}(\xi=0) & \sim-\epsilon \mathcal{S} w_{\xi}^{\text {outer }}(0) \\
& \sim-\epsilon^{2} \mathcal{S} \chi \\
& \sim-\epsilon^{2} \mathcal{S} \omega \zeta_{1}(\Xi)
\end{aligned}
$$

Thus,

$$
q_{20}=-\mathcal{S} \omega \zeta_{1}(\Xi)
$$

Recalling that $p_{20}=\omega \zeta_{0}(\Xi)$ and the matching constraint $p_{20}=q_{20}$ gives

$$
\mathcal{S}=-\zeta_{0}(\Xi) / \zeta_{1}(\Xi)
$$

Thus, we can determine the phase shift from the lowest-order inner approximation only. The coefficients $\zeta_{0}$ and $\zeta_{1}$ of the inner approximation are graphed in figure 2 .

In the limit $w(0) \rightarrow 0$, the elliptic modulus $k$ tends to one and the elliptic functions have but a single limiting peak so that the solutions are solitary waves. Unfortunately, the elliptic integral $K \rightarrow \infty$ so that the peaks are infinitely far from the origin in this limit. To obtain solitary wave solutions with peaks at a finite distance from $y=0$, we need to modify our assumptions about the parameters as done in the next section.
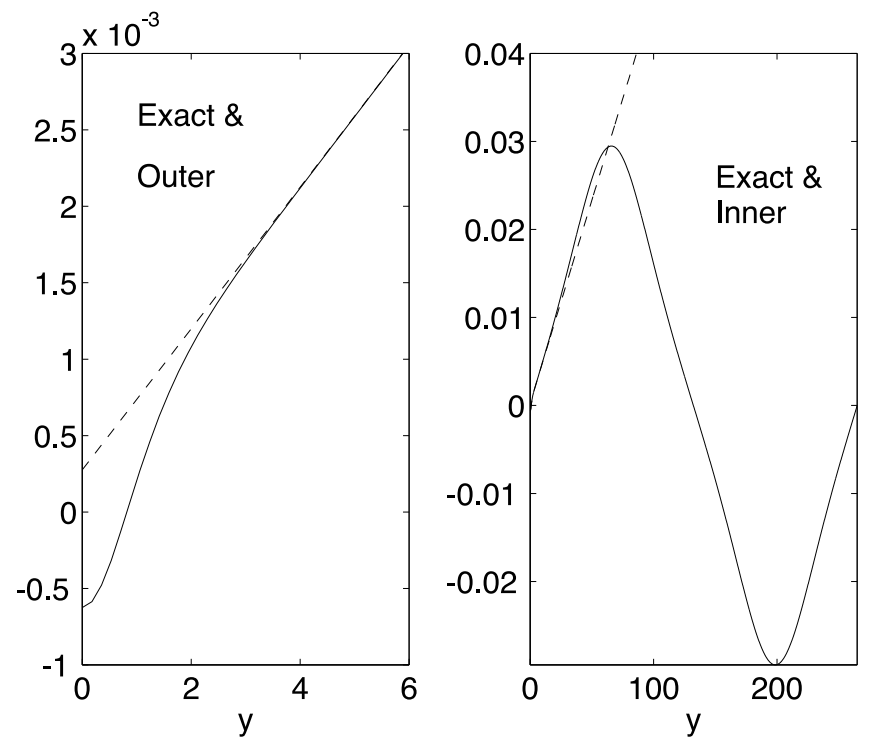

Figure 3. Two views of a solution whose outer solution is periodic rather than solitary. $\Xi=4$ and $w(0)=-\epsilon^{2}$, i.e., $\omega=-1$. Left: comparison of outer solution (dashed straight line) with the exact solution (solid). Right: same as left except that both the horizontal and vertical scale have been greatly expanded and the dashed line is the inner approximation. Because the solution is symmetric with respect to $y=0$, i.e., $w(-y)=w(y)$ for all $y$, only $y \geqslant 0$ is illustrated.

Figure 3 shows a typical nonsoliton example for $\epsilon=\frac{1}{40}$. The length scales of the inner and outer regions are so disparate that it is necessary to show each in a different panel. The left graph 
shows the breakdown of the outer approximation, which varies so slowly that it appears simply a straight line in the inner region. The right panel shows that the inner approximation, although a good approximation to the periodic elliptic function over a large range in $y$, eventually fails because it asymptotes to a straight line whereas the true solution oscillates slowly with $y$. Figure 4 shows the corresponding errors and the overlap region where both the inner and outer expansions are accurate, and can be matched to each other.

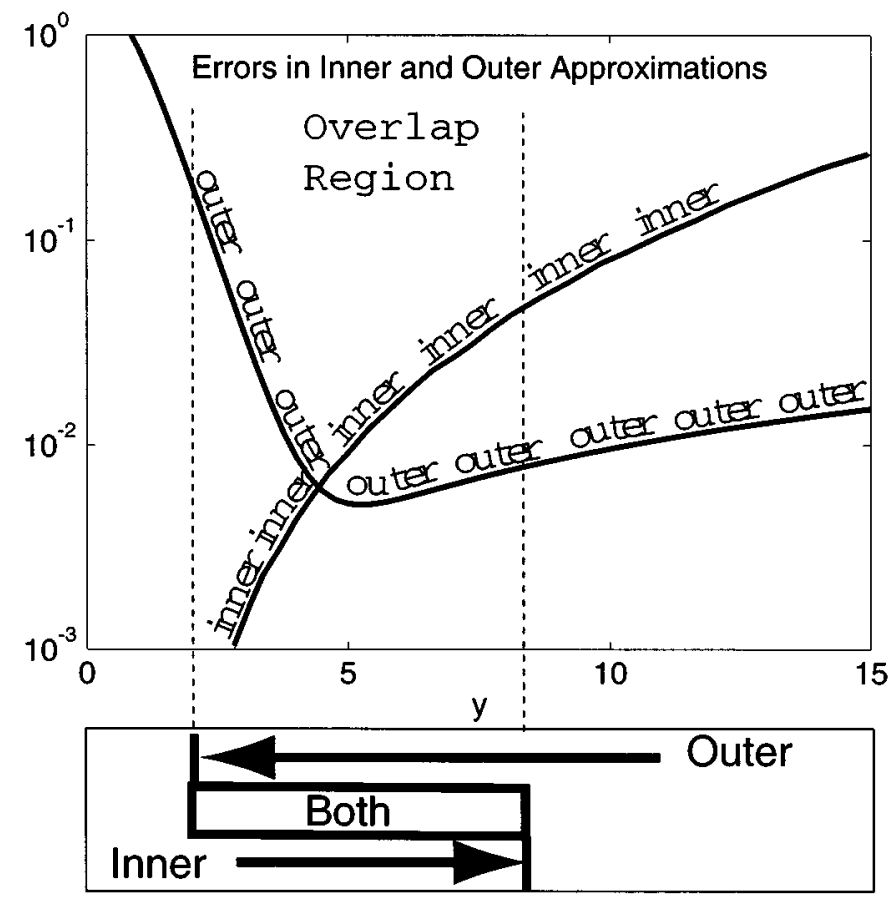

Figure 4. Same case as the previous figure, illustrating the errors of the inner and outer approximations versus $y$. The errors have been scaled by dividing by $w(0)$. The arrows at the bottom point to the limits of the validity of the inner or outer approximations. These two expansions have an overlap region, bounded by the vertical dotted lines, where both expansions are accurate, which allows the two approximations to be asymptotically matched. The outer approximation neglects only the term $\Xi \operatorname{sech}^{2}(y) w$ and is accurate for $|y| \gg 1 /|\log (\epsilon)|$. The lowest-order inner approximation neglects $-\epsilon^{2} w+2 w^{3}$ and is accurate for $|y| \ll 1 / \epsilon$.

\section{Analytic series expansions for the inner problem for $\Xi$ close to an integer}

When $\Xi$ is within $\mathrm{O}(\epsilon)$ of one of the special integer $\Xi$ where an exact solution is possible, an analytical solution in powers of $\epsilon$ is possible. Conveniently, this is precisely what is needed to match to an outer solution that is a soliton, and conversely, the solitary wave can match to the inner approximation only when $\Xi$ is near one of the analytically soluble $\Xi$. Thus, unlike the general case for arbitrary $\Xi$ where the second component $v$ or $w$ of the waves is periodic in space, it is not necessary to employ numerical methods to complete asymptotic matching for solitary waves. 


\subsection{Small $\Xi$ approximations}

For small $\Xi$, one can apply regular perturbation theory in powers of $\Xi$. A symbolic manipulation language like Maple is quite effective for performing the algebra if the Legendre equation is first written in terms of the coordinate $z$ : Maple is much better at manipulating powers and logarithms than in simplifying and combining the hyperbolic functions of the original coordinate $y$. The result is

$\Omega(y ; \Xi) \approx 1+\Xi\{-\log (\cosh (y))\}+\Xi^{2}\left\{-\frac{y^{2}}{2}+\log (\cosh (y))+\frac{1}{2}[\log (\cosh (y))]^{2}\right\}$.

Asymptotically, as $|y| \rightarrow \infty$,

$\Omega \sim\left\{1+\Xi \log (2)+\Xi^{2}\left(-\log (2)+\frac{1}{2} \log ^{2}(2)\right)\right\}+y\left\{-\Xi+(1-\log (2)) \Xi^{2}\right\}$.

\section{2. $\Xi$ near six}

The linear term in the outer limit is zero only when $\Xi=n(n+1)$ where $n$ is an even integer so that the exact solution is an ordinary Legendre function. To match to an outer solution that is a solitary wave, it is necessary that the linear term vanish to lowest order as will be explained below. Therefore, the next interesting value of $\Xi$ after zero is $\Xi=6$.

Set

$$
\Xi=6-5 \lambda \epsilon
$$

for some constant $\lambda$ which is $\mathrm{O}(1)$. This definition in effect changes the two independent parameters of a branch of solitary waves from $(\Xi, \epsilon)$ to $(\lambda, \epsilon)$. Expand the solution to the inner equation, equation (39), as

$$
\Omega(y ; \epsilon)=\Omega^{(0)}(y)+\epsilon \Omega^{(1)}+\epsilon^{2} \Omega^{(2)}(y)+\cdots
$$

subject to the initial conditions $\Omega(0)=1, \Omega_{y}(0)=0$. The exact solution for $\Xi=6$ gives

$$
\Omega^{(0)}=-2+3 \operatorname{sech}^{2}(y) .
$$

To solve the first-order equation, it is convenient to switch to $z=\tanh (y)$. One can easily verify through direct substitution that the solution is $\Omega^{(1)}=\lambda\left\{2 z^{2}-\frac{1}{2} \log \left(1-z^{2}\right)+\frac{3}{2} z^{2} \log (1-\right.$ $\left.\left.z^{2}\right)\right\}$ which when expressed in terms of the original coordinate is

$$
\Omega^{(1)}=\lambda\left\{2 \tanh ^{2}(y)+\log (\cosh (y))-3 \tanh ^{2}(y) \log (\cosh (y))\right\} .
$$

For large $y$, we find

$$
\Omega \sim-2+\epsilon \lambda 2(1+\log (2))+\epsilon \lambda(-2 y) .
$$

\section{Asymptotic matching, part II: solitons}

To approximate solitary waves, it is necessary that the outer solution should be a solitary wave:

$$
w^{\text {outer }}(\xi ; \epsilon)=\epsilon \operatorname{sech}(\xi-\mathcal{S}) .
$$

(Strictly speaking, this is the outer approximation for $\xi>0$; the approximation left of the origin is obtained by invoking the symmetry of the exact solution and its outer approximation: $w(y)=w(-y)$ for all $y$.) It is only necessary to explicitly match for $y>0$ because the symmetry ensures that the inner and outer approximation will also match for negative $y$.

Expanding the outer approximation as a power series in $\xi$ gives

$$
w^{\text {outer }}(\xi ; \epsilon) \approx \epsilon \operatorname{sech}(\mathcal{S})+\epsilon \operatorname{sech}(\mathcal{S}) \tanh (\mathcal{S}) \xi+\mathrm{O}\left(\xi^{2}\right)
$$


It follows that

$$
q_{10}=\operatorname{sech}(\mathcal{S}), \quad q_{11}=\operatorname{sech}(\mathcal{S}) \tanh (\mathcal{S}) .
$$

The matching conditions equation (57), $p_{10}=q_{10}, p_{20}=q_{20}$ and $p_{21}=q_{11}$ raise a difficulty: the match of $q_{10}$ requires a nonzero $\mathrm{O}(\epsilon)$ constant in the outer limit, but $q_{11}$ matches to a linear-in- $y$ term which is $\mathrm{O}(\epsilon)$ smaller. For general $\Xi$, however, the outer limit of the inner approximation has constant and linear-in- $y$ terms of equal magnitude.

The resolution of this conundrum is that solitary waves are restricted (for sufficiently small $\epsilon$ ) to neighbourhoods of those $\Xi$ where the linear-in- $y$ terms of the outer limit of the inner approximation are zero. This occurs when $\Xi=0,6,20,42, \ldots, 2 n(2 n+1)$, where $n$ is an integer, that is, whenever $\Xi$ is such that the inner problem can be solved exactly in terms of an ordinary Legendre polynomial in the coordinate $z=\tanh (y)$. We will analyse the neighbourhoods of $\Xi=0$ and $\Xi=6$ in turn.

\subsection{Small $\Xi$}

Define

$$
\Xi \equiv \lambda \epsilon
$$

where $\lambda$ is $O(1)$. This change shifts from $\Xi$ to $\lambda$ as an independent parameter.

The inner approximation is

$$
w^{\text {inner,lowest }}=\epsilon \omega \Omega(y ; \epsilon) .
$$

Note that in contrast to the previous section, the inner approximation is $\mathrm{O}(\epsilon)$ rather than $\mathrm{O}\left(\epsilon^{2}\right)$. The small- $\Xi$ approximations given in equation (67) are

$$
\Omega \sim\{1+\lambda \epsilon \log (2)\}+y\{-\lambda \epsilon\},
$$

which implies that coefficients of the outer limit of the inner approximation are

$$
p_{10}=\omega, \quad p_{11}=0, \quad p_{20}=\omega \lambda \log (2), \quad p_{21}=-\omega \lambda .
$$

The matching condition $p_{10}=q_{10}$ gives

$$
\omega=\operatorname{sech}(\mathcal{S}) .
$$

The matching condition $q_{11}=p_{21}$ gives

$$
\tanh (\mathcal{S})=-\lambda
$$

which implies that for $\Xi, \epsilon \ll 1$

$$
\mathcal{S}=-\operatorname{arctanh}(\lambda)=-\operatorname{arctanh}\left(\frac{\Xi}{\epsilon}\right) .
$$

The shift $\mathcal{S}$ is real only when

$$
|\lambda|<1 \Leftrightarrow|\Xi|<\epsilon .
$$

Thus, solitary waves exist (in the limit of small $\epsilon$ ) only in a fan-shaped region in the $\epsilon-\Xi$ plane radiating from the origin plus similar fans radiating from points on the $\Xi$-axis where $\Xi$ is equal to one of the other discrete special values where the inner approximation is a Legendre polynomial in $\tanh (y)$. 


\section{2. $\Xi$ near six}

The procedure is very similar to that for $\Xi \ll 1$. Define a new parameter $\lambda$ by

$$
\Xi=6-5 \lambda \epsilon \text {. }
$$

Using the large- $y$ asymptotics of the inner approximation for $\Xi \approx 6$, equation (72), the coefficients of the outer limit of $w^{\text {inner,lowest }}$ are

$p_{10}=-2 \omega, \quad p_{11}=0, \quad p_{20}=\omega \lambda 2(1+\log (2)), \quad p_{21}=-2 \lambda \omega$.

The matching condition $p_{10}=q_{10}$ gives

$$
\omega=-\frac{1}{2} \operatorname{sech}(\mathcal{S}) \text {. }
$$

The matching condition $p_{21}=q_{11}$ gives

$$
\mathcal{S}=\operatorname{arctanh}(\lambda) \Leftrightarrow \lambda=\tanh (\mathcal{S}) .
$$

The constraint $|\lambda|<1$ so that $\operatorname{arctanh}(\lambda)$ is real-valued implies that solitons are confined, to the extent that the perturbation theory is accurate, within a sector of the $\epsilon-\Xi$ plane bounded by the straight lines $\Xi=6 \pm 5 \epsilon$.

\section{Solitons and matching: numerical illustrations and discussion}

The easiest numerical way to calculate solitary waves is to specify $\epsilon$ and the shift of the soliton peak, $\mathcal{S}$. One can then numerically integrate from large positive or negative $y$ towards the origin, initializing with the shifted solitary wave, and then varying $\Xi$ until $w_{y}(0)=0$. (Note that a smooth function which is symmetric with respect to the origin always has a zero first derivative there.)

The perturbation theory predicts that the contours of constant shift $\mathcal{S}$ will be straight lines in the $\epsilon-\Xi$ plane. Figure 5 shows some numerically-computed contours. The curve for $\mathcal{S}=0$, which is the most curved of those illustrated, is well-fit over the interval $\epsilon \in\left[0, \frac{1}{5}\right]$ by $\Xi(\epsilon ; \mathcal{S}=0) \approx 6+11 \epsilon^{2}$, which shows that the difference from theory is due to terms of higher order in $\epsilon$.

When $\mathcal{S}=0$, the resulting structure might be dubbed a 'punctured solitary wave'. The solution $w(y)$ is an ordinary soliton, $w \sim \epsilon \operatorname{sech}(\epsilon y)$, everywhere outside the inner region. There, instead of rising smoothly to a peak at $w=\epsilon$, the $\operatorname{sech}^{2}(y)$ term forces $w$ to curve sharply downward so that it becomes negative and has a minimum rather than a maximum at the origin.

Figure 6 illustrates a typical case. The composite perturbative expansion for $\mathcal{S}=0$ is the dotted line. Almost all the error can be removed by simply shifting the outer solution; the composite-expansion-with-shift is also plotted, but resembles the numerical solution so closely that it cannot be seen as a separate curve.

Figure 7 shows the same value of $\Xi$, but with $\epsilon$ reduced by a factor of four from the previous illustration. The 40:1 ratio between the scale of $u$, which supplies the $\operatorname{sech}^{2}$ term in the differential equation for $w$, and the $\mathrm{O}(1 / \epsilon)$ length scale of $w$ for large $y$, requires the use of two graphs with different scales (left, top and bottom). The upper right graph shows that the error in perturbation theory is very small for sufficiently small $\epsilon$.

When the shift $\mathcal{S}$ is positive, then the outer solution acquires the two-humped structure illustrated in figure 8. The region of rapid variation at the origin glues together two copies of an ordinary NLS, sech-type solitary wave. Without the cross-modulation term, i.e. the $\operatorname{sech}^{2}(y)$ term in the reduced problem, a pair of NLS solitary waves cannot form a bound state. 


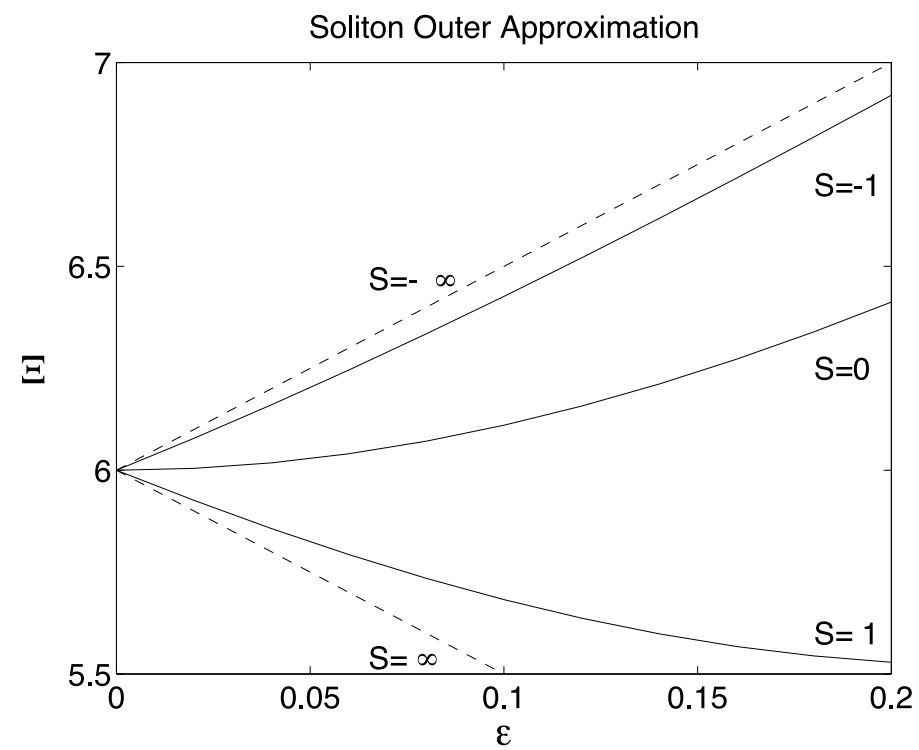

Figure 5. When the outer solution is a solitary wave with its peak shifted by $\mathcal{S}$ in the outer coordinate $\xi$, the perturbation theory predicts that solutions with a given $\mathcal{S}$ will be found only on the straight line $\Xi=6-5 \epsilon \tanh (\mathcal{S})$. The solid curves, which were numerically computed, show that the contours of constant $\mathcal{S}$ curve as $\epsilon$ increases, consistent with the increasing importance of higher-order terms. The dashed lines mark the perturbative (not numerical) limiting lines; matched asymptotics predicts that solitary waves are found only within the triangle-shaped sector bounded by these lines, and similar sectors radiating from other discrete points on the $\Xi$ axis.

(Note that for the nonlinear Schrödinger equation, the number of peaks is always either one or infinity, never a finite integer in between.)

The figure also clarifies another point that is worth reiterating. Analytically, the complete outer approximation is

$$
w^{\text {outer }}(y)= \begin{cases}\epsilon \operatorname{sech}(\epsilon y+\mathcal{S}), & y<0 \\ \epsilon \operatorname{sech}(\epsilon y-\mathcal{S}), & y>0 .\end{cases}
$$

If the outer approximation has a nonzero slope as $y \rightarrow 0$, then the function displayed in (88) will have a discontinuous slope at $y=0$ as shown by the $\mathrm{V}$-shaped dashed curve in figure 8 . The exact solution $w(y)$ is smooth and analytic because the outer approximation fails in the inner region where the extra $\operatorname{sech}^{2}(y)$ term turns $w$ rapidly from a negative slope just left of the origin to a positive slope of equal magnitude.

When $\mathcal{S}$ is negative, the peak of the outer solution is actually on the other side of the origin. When $\mathcal{S}$ is sufficiently large and negative, the outer approximation may be simplified to

$$
w^{\text {outer }} \sim\{2 \epsilon \exp (-|\mathcal{S}|)\} \exp (-\epsilon|y|), \quad \mathcal{S} \ll 0 .
$$

The maximum of the outer approximation is given by the constant in braces. When this is small compared with one, the nonlinear terms become negligible in the outer region. The approximation of neglecting the nonlinear terms for all $y$ in the reduced problem for $v$ has been dubbed the 'father-daughter' approximation because the $v$ component ('daughter') is very small in amplitude compared with the tall, sech-shaped peak in the $u$ component ('father'). As reviewed in [18], this 'father-daughter' approximation applies when

$$
\Xi=(2 n+\epsilon)(2 n+1+\epsilon) \approx 2 n(2 n+1)+(4 n+1) \epsilon .
$$




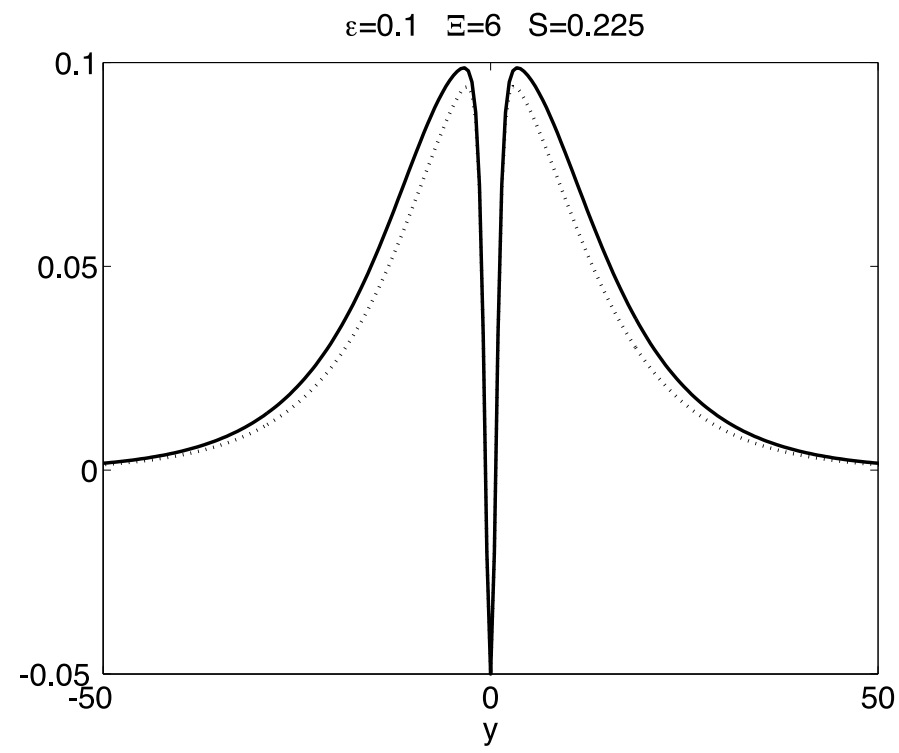

Figure 6. A 'punctured solitary wave'. The solid curve is the exact solution. The lowest-order composite expansion with the shift $\mathcal{S}=0.225$ is graphically indistinguishable from the numerical solution except at $y=0$ where the minimum in the perturbative approximation is about $10 \%$ too deep. The dotted curve is the perturbative approximation for $\mathcal{S}=0$; this does have a visible error, but this error can be almost completely removed merely by shifting the maxima of the sech-functions which are the outer approximation to $y= \pm \mathcal{S} / \epsilon$ with $\mathcal{S}=0.225$.

The case $n=1$ gives

$$
\Xi \approx 6+5 \epsilon, \quad \text { ['father-daughter linear approximation'] }
$$

which is simply the boundary line for solitons as predicted by the composite theory. The father-daughter approximation applies in the neighbourhood of the line $\mathcal{S}=-\infty$ (figure 5). The everywhere-linear-differential-equation for $v$ approximation is thus, for small $\epsilon$, merely a limiting case of the matched asymptotics theory.

\section{Explicit composite solution}

In this section, we collect our scattered approximations in one place. Our coupled boundaryvalue problem is the SOCNLS equations:

$$
\begin{aligned}
& u_{x x}-\mu^{2} u+\frac{\sigma_{1}}{\alpha_{1}} u^{3}+\frac{v_{12}}{\alpha_{1}} u v^{2}=0 \\
& v_{x x}-\beta^{2} v+\frac{\sigma_{2}}{\alpha_{2}} v^{3}+\frac{\nu_{21}}{\alpha_{2}} u^{2} v=0 .
\end{aligned}
$$

To zeroth and first order, the approximation for $u$ is, for both periodic waves and solitary waves,

$$
u \sim \sqrt{2 \frac{\alpha_{1}}{\sigma_{1}}} \mu \operatorname{sech}(\mu x)+\mathrm{O}\left(\epsilon^{2}\right) .
$$

The rather complicated solution for $v$ is most easily described by introducing the new coordinate and unknown

$$
y \equiv \mu x, \quad w=\sqrt{\frac{\sigma_{2}}{2 \alpha_{2} \mu^{2}}} v
$$



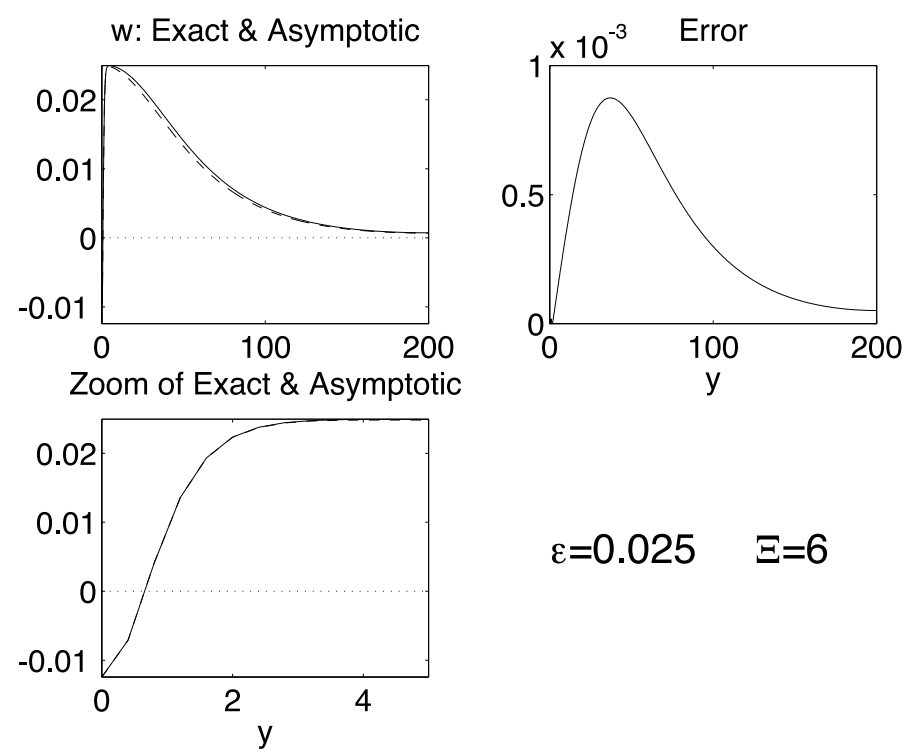

$$
\varepsilon=0.025 \quad \Xi=6
$$

Figure 7. Same as figure $6(\Xi=6)$ but for $\epsilon$ reduced to $\frac{1}{40}$. The two graphs on the left both show the solitary wave compared with the asymptotic approximation, but on different scales both for $w$ and $y$. The trough at $y=0$ is so narrow for small $\epsilon$ that it is invisible when graphed on the length scale of the outer solution, as shown in the upper left panel. The graph on the right illustrates the error: at worst, the difference between the composite expansion and exact solution is 28 times smaller (i.e. $\mathrm{O}(1 / \epsilon)$ smaller) than the maximum of $w$.

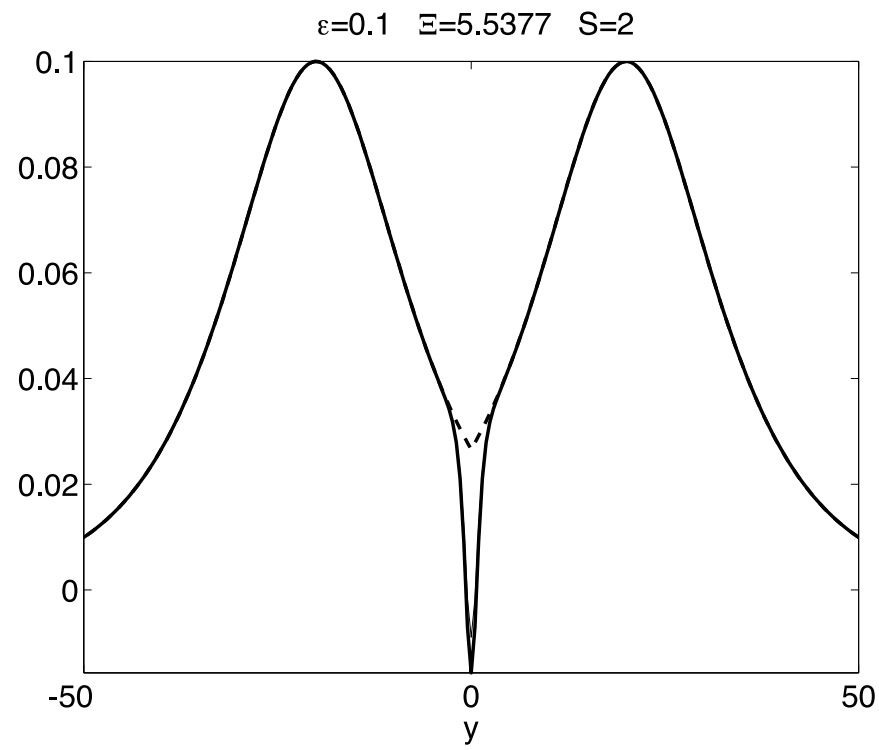

Figure 8. Solid curve: a two-humped solitary wave for $\epsilon=\frac{1}{10}$ and $\Xi=5.5377$. The outer approximation is shown as the dashed curve, which is distinguishable from the exact solution only in a narrow region around the origin. The outer approximation is a sech-function with a peak at $y=\mathcal{S} / \epsilon$ plus its mirror image where $\mathcal{S}=2$. 
and the new parameter

$$
\Xi \equiv 2 \frac{\nu_{21}}{\sigma_{1}} \frac{\alpha_{1}}{\alpha_{2}}
$$

The composite approximation for $w(y)$ is

$$
w^{\text {composite }} \sim w^{\text {inner }}+w^{\text {outer }}-\operatorname{outer} \operatorname{limit}\left(w^{\text {inner }}\right) .
$$

Periodic waves. In this case, $\Xi$ can be arbitrary. The inner approximation $w^{\text {inner }}$ must be computed numerically by the spectral algorithm described earlier. The numerical solution determines two functions which vary only with the parameter $\Xi$ and are independent of everything else, $\zeta_{0}(\Xi)$ and $\zeta_{1}(\Xi)$. The outer approximation is then an elliptic function which is

$$
w^{\text {outer }}=\epsilon k\left(1+4 \chi^{2}\right)^{1 / 4} \operatorname{cn}\left(\left(1+4 \chi^{2}\right)^{1 / 4}\left(\epsilon\left\{y+\zeta_{0}(\Xi) / \zeta_{1}(\Xi)\right\}\right)-K ; k\right)
$$

where

$$
\chi=\omega \zeta_{1}(\Xi)
$$

and the elliptic modulus $k$ is

$$
k=\sqrt{\frac{1}{2}+\frac{1}{2} \frac{1}{\sqrt{1+4 \chi^{2}}}}
$$

with $K(k)$ as the usual complete elliptic integral. The periodic waves are a three-parameter family where the parameters are $(\Xi, \epsilon, \omega)$ where $\omega \equiv w(0) / \epsilon^{2}$ and where $\Xi$ and $\epsilon$ are coefficients of the differential equation that cannot be eliminated by rescaling.

Solitary waves, case $I:|\Xi| \leqslant \epsilon$.

$w^{\text {inner }} \sim \epsilon \operatorname{sech}(\operatorname{arctanh}(\Xi / \epsilon))[1+\Xi\{-\log (\cosh (y))\}$

$$
\left.+\Xi^{2}\left\{-\frac{y^{2}}{2}+\log (\cosh (y))+\frac{1}{2}[\log (\cosh (y))]^{2}\right\}\right]
$$

$w^{\text {outer }} \sim \epsilon \operatorname{sech}\{\epsilon y+\operatorname{arctanh}(\Xi / \epsilon)\}$

outer $\operatorname{limit}\left(w^{\text {inner }}\right) \sim \epsilon \operatorname{sech}\{\operatorname{arctanh}(\Xi / \epsilon)\}\{1+\Xi \log (2)-y \Xi\}$.

Solitary waves, case II: $|\Xi-6| \leqslant 5 \epsilon$.

$$
\begin{aligned}
& w^{\text {inner }} \sim-\frac{1}{2} \epsilon \operatorname{sech}\left\{\operatorname{arctanh}\left(\frac{6-\Xi}{5 \epsilon}\right)\right\}\left[-2+3 \operatorname{sech}^{2}(y)\right. \\
& \left.+\frac{6-\Xi}{5}\left\{2 \tanh ^{2}(y)+\log (\cosh (y))-3 \tanh ^{2}(y) \log (\cosh (y))\right\}\right] \\
& w^{\text {outer }} \sim \epsilon \operatorname{sech}\left\{\epsilon y-\operatorname{arctanh}\left(\frac{6-\Xi}{5 \epsilon}\right)\right\} \\
& \text { outer } \operatorname{limit}\left(w^{\text {inner }}\right) \sim-\frac{1}{2} \epsilon \operatorname{sech}\left\{\operatorname{arctanh}\left(\frac{6-\Xi}{5 \epsilon}\right)\right\} \\
& \times\left\{-2+\left(\frac{6-\Xi}{5}\right) 2(1+\log (2))-2\left(\frac{6-\Xi}{5}\right) y\right\} .
\end{aligned}
$$

The solitary waves are a collection of two-parameter families which exist only within a sector that radiates from the point $\epsilon=0, \Xi=2 m(2 m+1)$ where $m$ is a non-negative 
integer; the $m=0$ and $m=1$ families are displayed above. The parameters can be taken as $(\Xi, \epsilon)$, provided the values lie within the sector $-|\Xi| \leqslant \epsilon$ for the lowest family, for example. Alternatively, one may specify $\epsilon$ and the shift $\mathcal{S}$ of the outer solution, which is more convenient for specifying numerical solutions. For the lowest branch, $\mathcal{S}=-\operatorname{arctanh}(\Xi / \epsilon)$; for the solitary waves with $\Xi \approx 6, \mathcal{S}=\operatorname{arctanh}((6-\Xi) / 5 \epsilon)$ and similarly for other branches.

\section{Summary}

In this work, we have shown that one branch of solitary waves of the CNLS system can be approximated analytically using the method of matched asymptotic expansions. Previous authors who found this branch numerically, such as $[2,10]$, were pessimistic that such approximations would ever be found because the solitary wave is intrinsically nonlinear for large $|y|$ and cannot be approximated uniformly in space by an everywhere-linear differential equation. Matched asympotics expansions are untroubled by the nonlinearity of the outer approximation because the outer equation is analytically soluble in elliptic functions or the hyperbolic secant function.

Previous numerical studies [25] suggest that these solitary waves are unstable. However, a thorough stability study has not yet been performed.

One virtue of this work is that the method of matched asymptotic expansions is very general. We conjecture that it should be applicable to other solitary wave problems where the solitons have multiple spatial scales. (We confess that we have not yet identified further targets for the applications of matched asymptotics.)

However, Haelterman and Sheppard [10] have shown that there are additional solution branches with more maxima and minima than in the solitons discussed here. It seems likely that matched asymptotics would be fruitful for these branches, too.

Another significance of our perturbative approximations is that one general strategy for numerical computation of solitary waves is to discretize the differential equation and apply a Newton iteration. The continuation method can supply the required starting point for the iteration: as some parameter such as the frequency ratio $\epsilon$ is increased in small steps, the solution for one value of $\epsilon$ can be used as the first guess for a slightly larger $\epsilon$. In this way, one can trace a complete branch of solutions. The only problem is that some other approximation must be used to initialize the continuation method itself. The matched asymptotics approximation for small $\epsilon$ can supply such a starting point.

The most interesting generalization of our work is to apply matched asymptotics to predict new species of solitary waves for other wave systems. Another generalization is to look for unsymmetric solitary waves of the CNLS system.

\section{Acknowledgments}

This work was supported by the National Natural Science Foundation of China through grant 49375239 and by the American National Science Foundation through grant OCE9521133. We thank the referee for detailed and helpful comments.

\section{References}

[1] Agraval G P 1995 Nonlinear Fiber Optics 2nd edn (Boston, MA: Academic)

[2] Akhmediev N and Ankiewicz A 1993 Novel soliton states and bifurcation phenomena in nonlinear fibre couplers Phys. Rev. Lett. 70 2395-8

[3] Berry M V and Howls C J 1993 Infinity interpreted Phys. World 6 35-9 
[4] Boyd J P 1986 Polynomial series versus sinc expansions for functions with corner or endpoint singularities $J$. Comput. Phys. 64 266-9

[5] Boyd J P 1987 Spectral methods using rational basis functions on an infinite interval J. Comput. Phys. 69 112-42

[6] Boyd J P 1989 The asymptotic Chebyshev coefficients for functions with logarithmic endpoint singularities Appl. Math. Comput. 29 49-67

[7] Boyd J P 1998 Weakly Nonlocal Solitary Waves and Beyond-All-Orders Asymptotics: Generalized Solitons and Hyperasymptotic Perturbation Theory (Mathematics and Its Applications vol 442) (Amsterdam: Kluwer) p 608

[8] Boyd J P 1999 Chebyshev and Fourier Spectral Methods 2nd edn (New York: Dover) at press

[9] Boyd J P 1999 The Devil's invention: asymptotics, superasymptotics and hyperasymptotics Acta Appl. 56 1-98

[10] Haelterman M and Sheppard A P 1994 Bifurcation phenomena and multiple soliton-bound states in isotropic Kerr media Phys. Rev. E 49 3376-81

[11] Hasegawa A and Kodama Y 1995 Solitons in Optical Communication (Oxford: Clarendon)

[12] Manakov S V 1973 On the theory of two-dimensional stationary self-focusing of electromagnetic waves Sov. Phys.-JETP 38 248-53

[13] Menyuk C R 1988 Stability of solitons in birefringent optical fibres. II. Arbitrary amplitudes J. Opt. Soc. Am. B 5 392-402

[14] Paris R B and Wood A D 1995 Stokes phenomenon demystified IMA Bull. 31 21-8

[15] Pulov V I, Uzunov I M and Chacarov E J 1998 Solutions and laws of conservation for coupled nonlinear Schrödinger equations: Lie group analysis Phys. Rev. E 57 3468-77

[16] Segur H, Tanveer S and Levine H (ed) 1991 Asymptotics Beyond All Orders (New York: Plenum) p 389

[17] Tan B 1996 Collision interactions of envelope Rossby solitons in a barotropic atmosphere J. Atmos. Sci. 53 1604-16

[18] Tan B and Boyd J P 1999 Coupled-mode envelope solitary waves in a pair of cubic Schrödinger equations with cross modulation: analytical solution and collisions Chaos, Solitons Fractals at press

[19] Tan B and Liu S 1995 Collision interactions of solitons in a baroclinic atmosphere J. Atmos. Sci. 52 1501-12

[20] Tratnik M V and Sipe J E 1988 Bound solitary waves in a birefringent optical fibre Phys. Rev. A 38 2011-17

[21] Uzunov I M, Muschall R, Gölles M, Kivshar Y S, Malomed B A and Lederer F 1995 Pulse witching in nonlinear fibre directional-couplers Phys. Rev. E 51 2527-37

[22] Van Dyke M 1975 Perturbation Methods in Fluid Mechanics 2nd edn (Stanford, CA: Parabolic)

[23] Yang J 1996 Coherent structures in weakly birefringent nonlinear optical fibres Stud. Appl. Math. 97 127-48

[24] Yang J 1996 Vector solitons and their internal oscillations in birefringent nonlinear optical fibres Stud. Appl. Math. 98 61-97

[25] Yang J 1997 Classification of the solitary waves in coupled nonlinear Schrödinger equations Physica D 108 92-112

[26] Yang J 1998 Multiple permanent-wave trains in nonlinear systems Stud. Appl. Math. 100 127-52

[27] Yang J and Benney D J 1996 Some properties of nonlinear wave systems Stud. Appl. Math. 96 111-39

[28] Zakharov V E and Schulman E I 1982 To the integrability of the system of two coupled nonlinear Schrödinger equations Physica D 4 270-4 